\title{
Short educational programs in optical design and engineering
}

\section{Anna Voznesenskaya, Galina Romanova, Alexey Bakholdin, Nadezhda Tolstoba, Kseniia Ezhova}

Anna Voznesenskaya, Galina Romanova, Alexey Bakholdin, Nadezhda Tolstoba, Kseniia Ezhova, "Short educational programs in optical design and engineering," Proc. SPIE 9946, Optics Education and Outreach IV, 994619 (27 September 2016); doi: 10.1117/12.2237181

SPIE Event: SPIE Optical Engineering + Applications, 2016, San Diego, California, United States 


\title{
Short educational programs in Optical Design \& Engineering Anna Voznesenskaya, Galina Romanova, Alexey Bakholdin, Nadezhda Tolstoba, Ksenia Ezhova ITMO University (Russian Federation)
}

\begin{abstract}
Globalization and diversification of education in optical engineering causes a number of new phenomena in students' learning paths. Many students have an interest to get some courses in other universities, to study in international environment, to broaden not only professional skills but social links and see the sights as well etc. Participation in short educational programs (e.g. summer / winter schools, camps etc.) allows students from different universities to learn specific issues in their or in some neighbor field and also earn some ECTS for the transcript of records. ITMO University provides a variety of short educational programs in optical design \& engineering oriented for different background level, such are: Introduction into optical engineering, Introduction into applied and computer optics, Optical system design, Image modeling and processing, Design of optical devices and components. Depending on students' educational background these programs are revised and adopted each time. Usually the short educational programs last 4 weeks and provide 4 ECTS. The short programs utilize a set of out-of date educational technologies like problem-based learning, case-study and distance-learning and evaluation. Practically, these technologies provide flexibility of the educational process and intensive growth of the learning outcomes. Students are satisfied with these programs very much. In their feedbacks they point a high level of practical significance, experienced teaching staff, scholarship program, excellent educational environment, as well as interesting social program and organizational support.
\end{abstract}

Keywords: short educational program, optical education, optical engineering, optical design

\section{INTRODUCTION}

Short educational programs (SEP) are an excellent opportunity to deep knowledge in the professional area as well as advance communicative skills. Positive effort from the education in an international medium is shown in many publications, e.g. [1]. These programs are oriented on best practices of leading world universities and provide considerable learning outcomes by means of high quality of the education. Besides, SEPs sharpen each participant's ability to succeed in a rapidly changing world [2].

The reasons students seek SEP are numerous, and grow constantly [3]. Examples of students' motivation to enter a SEP are in its practical convenience: allowance to keep tightly scheduled degree plans and job responsibilities; lack of willing to study abroad for a long time.

In most cases SEP are devoted to broadening cross-cultural and communicative links between countries, universities and students. Meantime, rare professional SEPs in R\&D exist as well. The latter are more complex due to necessity to adopt each time the content and educational technologies to a certain students' group.

As pointed in [3] within higher education, the definition of SEPs is variable. They could be year-long, semester, twomonth module and less. The most effective SEPs last a month and could involve lectures, discussions, readings, interactive problem-solving, or laboratory works.

Short-term programs at ITMO University offer an opportunity to dive right into area of interest or related area and connect with an international group of peers, adding some serious rocket fuel to education, research and career opportunities [4].

In this paper four-weeks SEPs in Optical Design \& Engineering are described including Introduction into optical engineering, Introduction into applied and computer optics, Optical system design, Image modeling and processing, Design of optical devices and components.

Optics Education and Outreach IV, edited by G. Groot Gregory, Proc. of SPIE Vol. 9946, 994619

(C) 2016 SPIE · CCC code: $0277-786 X / 16 / \$ 18 \cdot$ doi: $10.1117 / 12.2237181$ 


\section{OVERVIEW OF THE SHORT EDUCATIONAL PROGRAMMS}

The proposed SEPs are purposed firstly for international students. In this relation effective learning time distribution is one of the main issues. Here, on the one hand SEP has to provide a considerable load of practical studies, on the other hand the contact studies have to be squeezed due to lodging and financial circumstances. Such a way SEPs consist of two parts - online training (2-3 weeks) and contact studies at ITMO University (1-2 weeks). The online training module is devoted to theory studies and knowledge assessment, while the contact studies module is directed on practical tasks and projects.

SEPs are realized at Department of Applied and Computer Optics [5], ITMO University in collaboration with Students' research laboratory for optical engineering [6]. Several different summer camps provide material for the students with different background and different individual learning paths.

\section{Introduction into optical engineering}

This SEP includes lectures and labs devoted to basics of optical engineering.

Key modules:

- Optical design fundamentals

- Basic optical elements

- Mounting of optical components

List of projects:

- Calculation of two-component system.

- The ray beams constraints.

- Calculation of telescopic system.

- Aberrations of the optical system.

- Study of the optical elements. Determination of optical parameters.

- Study of the optical elements. Determination of design parameters.

- Study of the optical elements. Determination of technological parameters.

- Optical lenses.

- Optical prisms.

- Ray tracing in optical system.

- Statement of experience in a laboratory simulation of total internal reflection.

\section{Introduction into applied and computer optics}

This SEP includes lectures and labs on basics of applied and computer optics, it discusses the role of optical design software on each stage of optical engineering including the stage of choosing the principal scheme up to the image processing.

Key modules:

- Properties and features of various optical elements used in optical devices and units.

- Basic principles of mounting of the optical elements and units.

- Imaging optical systems.

- Image processing for various purposes.

List of projects:

- Design of an optical system for laser beam concentration. 
- Design of the photoobjective with fixed focal length - Petzval scheme.

- Ray tracing in conic lightguides.

- Fiber optic sensor modeling.

- Ray tracing in graded index structures.

- Development of fastening the optical system of a laser beam expander.

- Design of the prism fastening.

- Preparation of images for observing through the raster.

- Determining the fringe sag during the interferogram decoding.

- Determining the similarity degree between different orientation images.

\section{Optical system design}

This SEP incorporates a list of 13 topics for optical system design. It includes some basic theory and practical skills of using special optical design software, and also an experience that can be applied for developing a wide range of optical instruments and devices.

Key modules:

- Basics of aberration theory. Types of aberrations. Chromatic and monochromatic aberrations.

- Mathematical description of aberrations. Seidel sums. Paraxial angles, paraxial heights.

- Theory of synthesis of optical systems. Algebraic method. Composing method.

- Mathematical basis of optimization. Local optimization. Merit Function.

- Evaluation of the image quality. Diffraction limited quality. Geometrical image quality. Criteria of the image quality. Strehl ratio. Rayleigh criterion. RMS criterion. Point spread function. Modulation transfer function. Encircled energy.

List of projects:

- Design of a two-mirror optical scheme of a telescope's objective.

- Design of a catadioptric scheme of a telescope's objective.

- Design a telescopic system with lens inverting system.

- Design of a telescopic system with prism inverting system.

- Design of a microscope's objective.

- Design of a system for laser beam collimation.

- Design of an objective for the LWIR region.

- Design of a condenser lens.

- Design of an eyepiece.

- Design of a microscope's illumination system in microscope.

- Design of a lens endoscopic system.

\section{Optical image modeling and processing}

This SEP includes lectures and labs devoted to optical imaging theory and basic principles of image processing. Projects include optical image modeling and optical image processing in $\mathrm{C}++$ programming language. 
Key modules:

- Basics of electromagnetic theory. Maxwell's equations and the wave equation. Imaging theory. Diffraction.

- Mathematical description and algorithms of image forming at coherent, incoherent and partial coherent illumination.

- Image quality parameters. Aberrations. PSF. MTF.

- The simulation of the image formation in coherent, incoherent and partially coherent light.

- Modeling of the influence of various factors on the optical image and its quality.

- Digital optical image registration and coding. Basics digital image processing.

- Geometrical, arithmetic and logical image transformations. Image filtering and image recovery.

\section{Design of Optical Devices and Components}

This summer camp includes lectures and labs devoted principles of design of optical devices. Basic working methods of the development of optical instruments in the modern production are considered. The focus is on the design and development of device units. The effective work in CAD systems is also considered.

Key modules:

- Mounting of lenses.

- Multiple-component lens assemblies.

- Mounting of optical windows, filters, shells, and domes.

- Techniques for mounting of prisms.

- Techniques for mounting of mirrors (nonmetallic, metallic).

- Estimation of mechanical stresses.

- Effects of temperature changes.

- The optical device's workflow.

- The arrangement of optoelectronic devices.

- Calculation and selection of basic parameters of optical devices.

- Development of drawings for optical components and mounting components.

- Development of routing device assembly site.

\section{BASIC MODULES FOR THE SHORT EDUCATIONAL PROGRAMS}

Presented SEPs embrace various spheres of optical engineering. As can be seen from the short description, a SEP can be easily adopted for different educational background and desirable specializations. On the figure 1 the basic modules are shown which can be composed to the desired course.

The module Applied Optics can be varied: it usually includes some information regarding standard optical elements and their typical application in optical devices. For the most students it is a theoretical module they know at least partly, in basics or can easily find in text-books which are available in every language. That is why this part of the course is most often is in on-line part of the SEP.

Optical system design module includes both basics of optical system design and more specified parts intended for those who would like to specialize in that area. Therefore, the basics of optical system design are often included into introductory courses: students have to know not only the first-order properties of different optical elements but they also 
have to know aberration properties and have the basic ideas about why optical systems for given optical devices, units and instruments have some special structure and features.

Modules:

Programs:

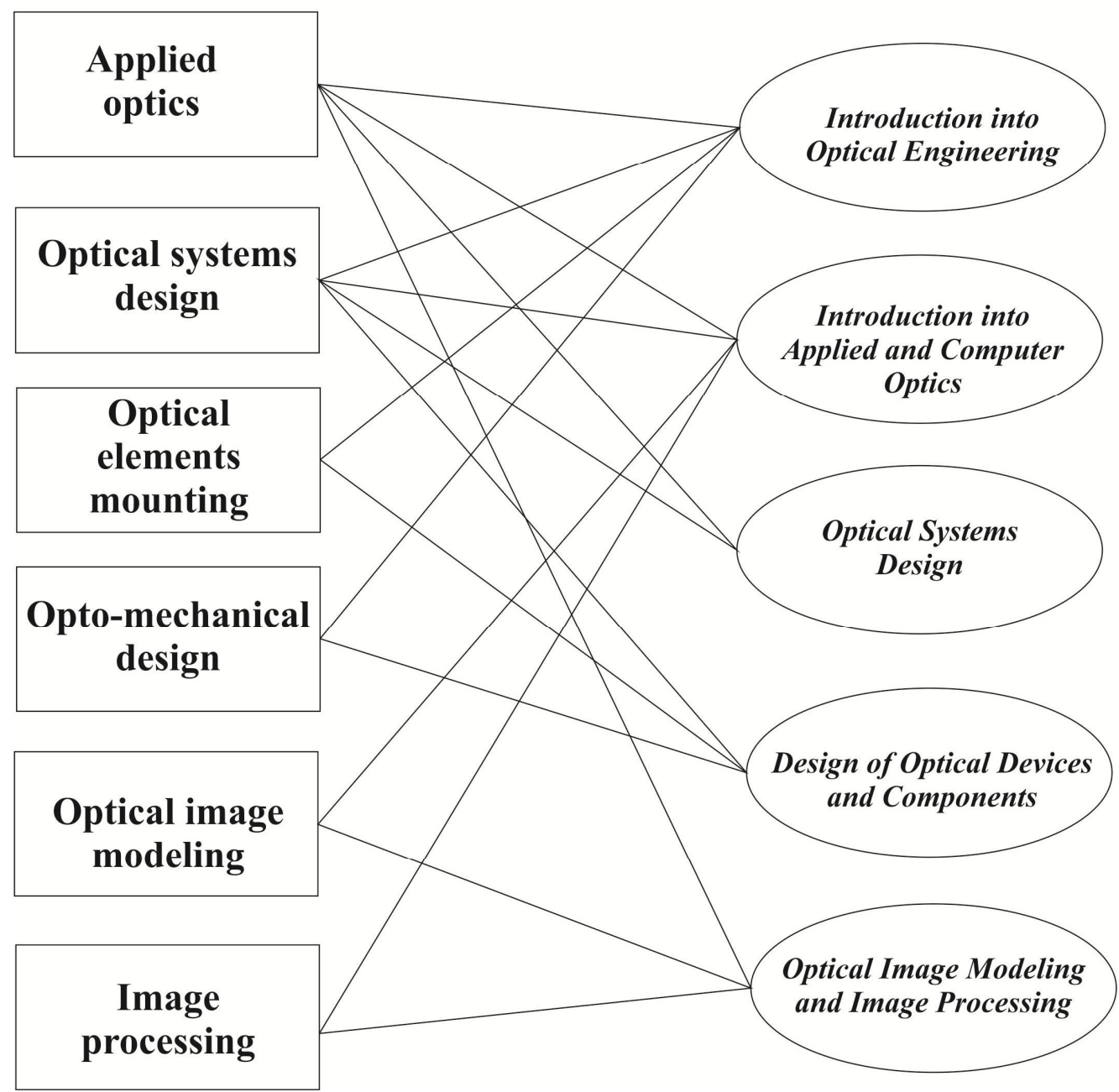

Figure 1 Modules and programs

Optical Elements Mounting is a very useful module, it helps to understand rules and principles of optical mounting and mechanical design. Usually when becoming familiar with this area students have the possibility to deal with the elements of real (physical) parts of optical instruments and / or have to design their own simple mechanical design. This course also helps an optical designer to understand correlation between the optical details and the tolerancing, and mechanical design.

Opto-mechanical Design module includes both brief overview of element mounting and optical systems mounting using various methods.

Optical Image Modeling module deals mostly with mathematical description of optical phenomena and elements modeling, it also helps to understand deeply how the optical design software works and provides the basic knowledge for those who would like to develop optical software for various purposes.

Image Processing module deals with principles of image registration, coding, filtering and other transformation. 
On the figure 1 only several possible courses with the included modules are shown, but this module structure can help compose new courses and programs, which can be easily adopted to the students. Moreover, this module structure may be a basis for graduate educational program development.

\section{FORMS OF THE SHORT EDUCATIONAL PROGRAMS}

Depending of the desired time to spend in St. Petersburg a SEP can include only 1 week of on-site training, the rest time are on-line studying and time for the certain project. This variant includes very intensive on-site training with nearly all necessary material for working on project. During the on-site training students are also offered some topics for projects they have to prepare. The assigned supervisor gives the initial task and describes steps to prepare the project. A supervisor also answers the questions via e-mail or Skype and gives the recommendation regarding the final report. Usually the report contains the initial data, description of stages for implementation of the project and comments on the results and conclusion. This form of SEPs suits well for PhD students and also for advanced students (master students), because it requires high-level of motivation and much self-work.

For most cases SEPs includes 2 weeks for on-line training. The on-line part includes 2 modules for two weeks, theoretical (informational) case can be used as many times as needed, the evaluation (assessment) part includes tests with both theoretical questions and practical tasks and only 3 trials to pass it. After the successful passing this part students come to on-site training. During two on-site weeks they have both lectures with theoretical material and practical classes, usually on computers with special software for optical system modeling, mechanical design, etc. Also during these weeks they have some time to prepare the projects. On the last day of the on-site training they have to present they project. During the presentation they answer the questions of the teacher, lecturer and other students and get recommendations and comments. Figure 2 shows students during the classes; figure 3 presents fragments of students' projects.
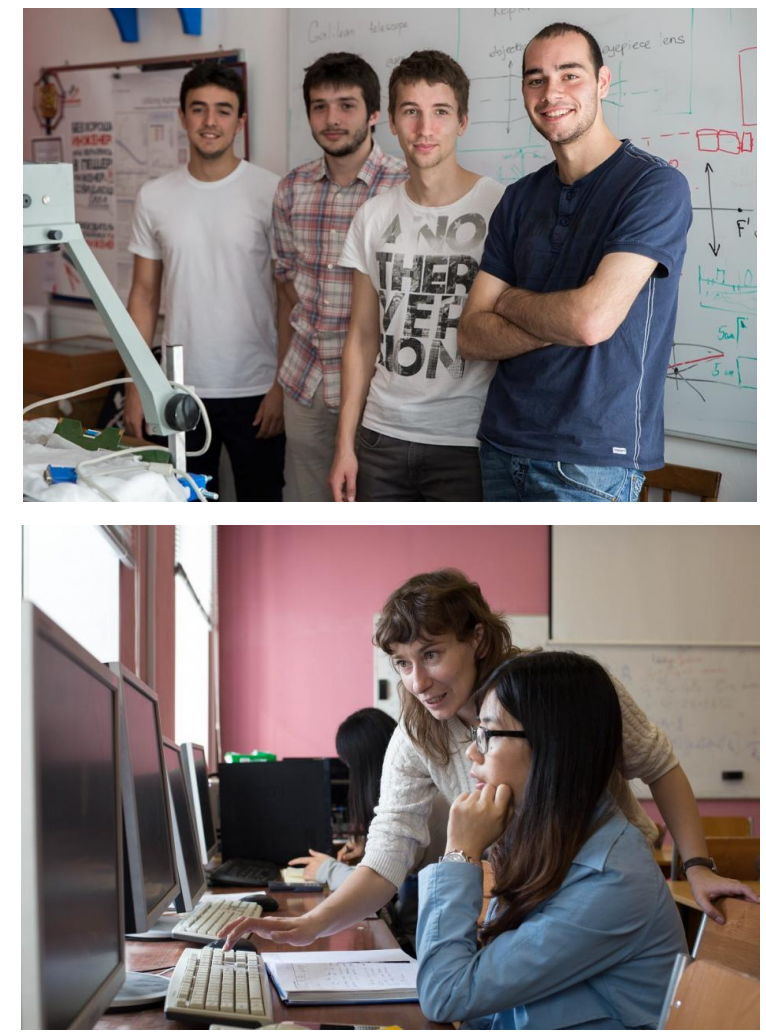
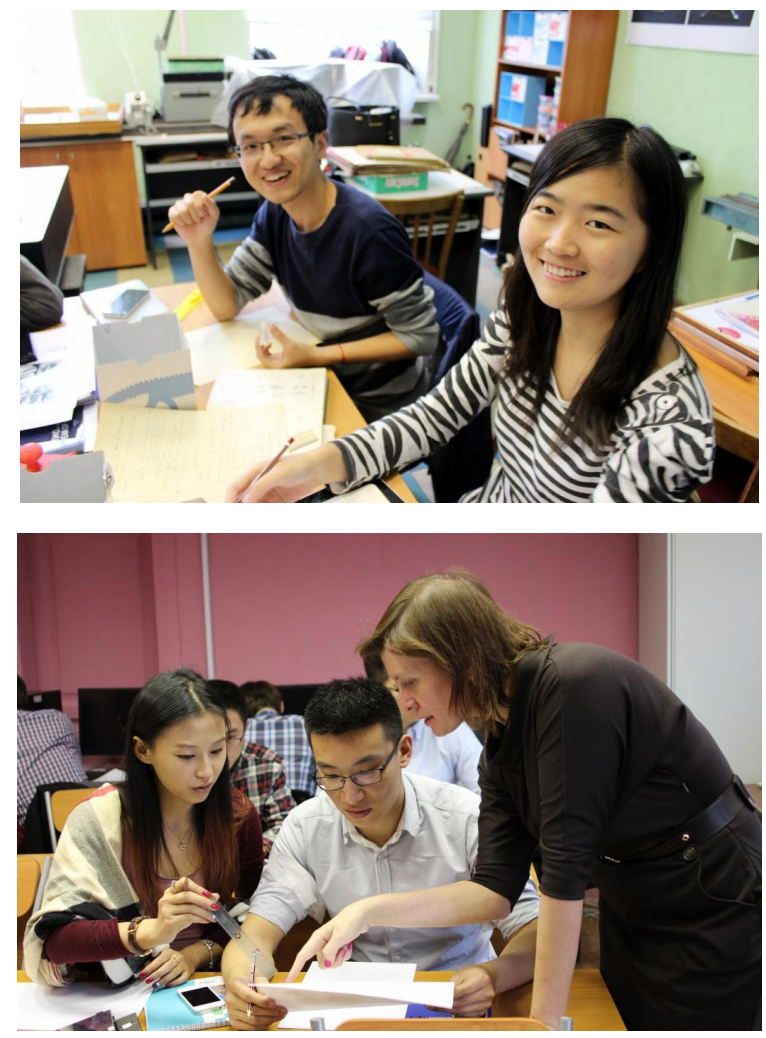

Figure 2 Students during the classes 


\section{Choice of the eye-piece}

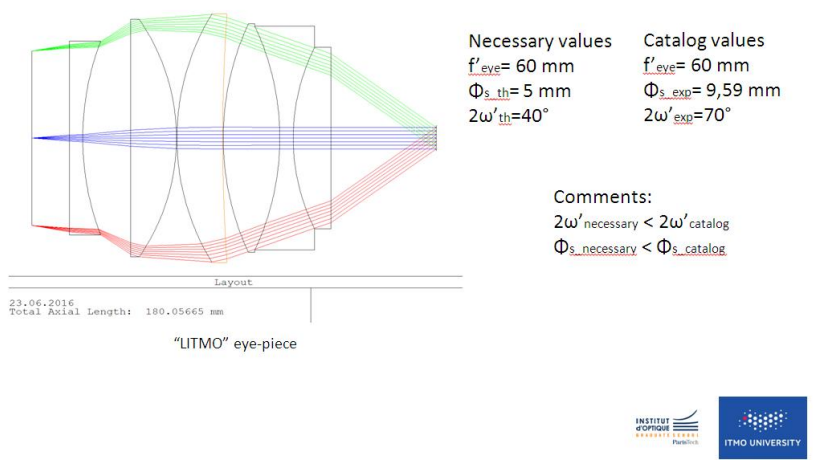

\section{Correction of the Objective}

- We set radii and conics of both mirrors as variables and use the Merit Function

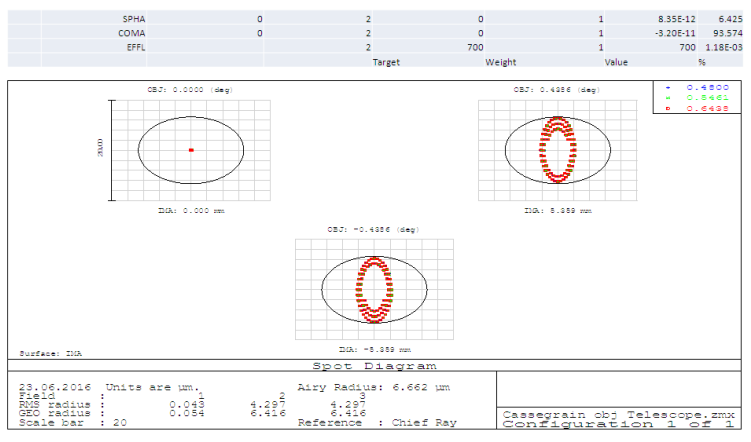

Figure 3 Fragments of the presentation prepared by students

\section{CONCLUSION}

Short educational programs are the useful form of organizing the classes for studying the topics and areas that are the best and know-how of the university. ITMO University offers several courses that include some basic modules on Applied optics, Optical systems design, Opto-mechanical Design, Image modeling and processing. These modules can be easily adopted and completed to desired specialization. Usually these courses take place in summer while international students have an ability to move. Such a way, students can have a good practice in neighboring academic field and a good chance to become familiar with Russian culture, St. Petersburg, and ITMO University - the center of optical education and science of Russian Federation. Therefore SEPs can be interesting for students from various universities which deal with optical engineering, design, development and application of various optical devices and instruments.

\section{REFERENCES}

[1] Bakholdin A., Kujawinska M., Livshits I., Styk A., Voznesenskaya A., Ezhova K., Ermolayeva E., Ivanova T., Romanova G., Tolstoba N. Double degree master program "Optical design" // Proceedings of SPIE. 2015, Vol. 9793, pp. 979307.

[2] MIT Professional Education. Available at http://professional.mit.edu/programs/short-programs/overview.

[3] Guide to Successful Short-Term Programs Abroad, 2nd Edition. Edited by Sarah E. Spencer and Kathy Tuma. NAFSA, Association of International Educators. 2007. P.246.

[4] Summer/Winter Schools. Available at http://en.ifmo.ru/en/page/296/Summer_Winter_School.htm.

[5] Department of Applied and Computer Optics, ITMO University. Available at: aco.ifmo.ru/education_eng.html.

[6] Tolstoba N.D., Saitgalina A.K., Abdula P.A., Butova D.V. Student research laboratory for optical engineering // Proceedings of SPIE. 2015, Vol. 9793, pp. 97931Y. 